\title{
Posterior Surgery for Cervical Myelopathy: Laminectomy, Laminectomy with Fusion, and Laminoplasty
}

\author{
John M. Rhee, Sushil Basra
}

The Emory Spine Center, Emory University School of Medicine, Atlanta, GA, USA

\section{Introduction}

Cervical myelopathy describes a constellation of symptoms and signs arising from compression of the cervical spinal cord (Table 1). Because the presentation of the myelopathic patient can be quite subtle in its early manifestations, the diagnosis may easily be missed or wrongly attributed as a "normal" epiphenomenon of aging. However, because the natural history is typically one of stepwise progression, early recognition and treatment is essential for optimal outcomes before the onset of irreversible spinal cord damage.

Considerable debate exists regarding the optimal surgical approach for treating multilevel cervical myelopathy ${ }^{1,2}$. Proponents of anterior surgery cite as advantages the ability to directly remove the majority of compressive pathologies encountered in the cervical spine (e.g., disc herniations, ventral osteophytes, osteophytes or ossification of the posterior longitudinal ligament [OPLL]), the muscle sparing dissection which results in minimal postoperative pain, and the ability to correct and decompress the cord over kyphotic lesions. Indeed, if myelopathy arises from one or two segments, the supremacy of an anterior approach is difficult to argue. However, when three or more segments are involved, accelerated complication rates associated with anterior surgery, particularly fusion related problems seen with long strut grafts used to reconstruct multilevel corpectomies, make posterior options more attractive.

Posterior based operations -- such as laminectomy, laminectomy and fusion, and laminoplasty -- possess their own distinct set of advantages. First, because an indirect decompression is performed, posterior surgeries are generally technically easier operations to perform than anterior corpectomies, particularly in multilevel patients with severe stenosis or OPLL that requires resection. Accordingly, all challenges associated with graft carpentry to reconstruct the anterior column are avoided. Second, posterior decompression allows the surgeon to rapidly decompress multilple segments more quickly than is possible with a multilevel anterior decompression. This may be critical in treating debilitated patients who need a quick decompressive procedure. Third, motion-preserving posterior operations like laminoplasty allow cord decompression without necessitating fusion and its attendant complications. Fourth, because fusion is not routinely necessary with some posterior approaches like laminoplasty, laminoplasty allows decompression of segments at future risk in one operation without substantially increasing patient morbidity. With a laminoplasty, a $\mathrm{C} 3$ to $\mathrm{C} 7$ decompression can be routinely performed with one operation, even if the majority of the stenosis is at, for example, $\mathrm{C} 4-7$, with a mild or moderate amount of stenosis at C3-4. In contrast, if an anterior approach were used in the same patient, one might hesitate to include a mildly/ moderately stenotic level at C3-4 for fear of increasing complications and morbidity, but then leave the patient vulnerable to subsequent disease at that adjacent level over time.

Posterior surgery is not appropriate in all myelopathic patients, however, and it clearly has its own set of draw-

Received Aug 8, 2008; accepted Aug 26, 2008

Corresponding author: John M. Rhee, MD

The Emory Spine Center 59 Executive Park South, Suite 3000

Atlanta, GA 30329, USA

Tel: +404-778-7021, Fax: +404-778-7117, E-mail: john_rhee@emoryhealthcare.org 
backs as well. It is associated with extensive posterior muscle denervation and a less cosmetically appealing scar. Additionally, as most of the compressive structures that lead to cervical myelopathy arise anteriorly, posterior-based procedures for spinal cord decompression rely on the ability of the cord to drift away from the anterior lesions as a result of releasing the posterior tethers (laminae, ligamentum flavum). Although such drift back reliably occurs in a lordotic or neutral cervical spine, it may not occur in the setting of significant kyphosis. Thus, the indications for performing posterior decompression are limited to those in whom the overall sagittal alignment is conducive to cord drift-back. In certain situations, posterior based operations for cervical myelopathy may not be sufficient, requiring anterior or combined anterior and posterior approaches (Fig. 1).

In this paper, we examine the roles of posterior decompression procedures-laminectomy, laminectomy and fusion, and laminoplasty-in the treatment of multilevel cervical myelopathy.

\section{Laminectomy alone}

Prior to the advent of anterior cervical spine surgery, laminectomy was the most common approach to decompression for multilevel myelopathy. Currently, however, laminectomy alone for the treatment of cervical myelopathy has been relegated to a relatively minor role due to its numerous downsides and the fact that better alternatives exist. Post-laminectomy kyphosis can occur after laminectomy and lead to potential recurrent myelopathy if the cord becomes draped over the kyphos (Fig. 2). The true incidence in the adult population is unknown, but estimates range from 11 47\% $\%^{3,4}$. The kyphosis can not only be a source deformity but also neck pain from muscular fatigue. In a comparative study, 34\% of patients developed postoperative kyphosis or swan neck deformity after laminectomy, versus $7 \%$ after laminoplasty 5 . If an over-aggressive facetectomy is performed along with laminectomy, iatrogenic spondylolisthesis can occur and potentially lead to pain and neurologic compromise. In addition to deformity and instability, post-laminectomy membranes may develop postoperatively and can lead to dynamic compression of the spinal cord over time ${ }^{6}$. Even in the absence of a symptomatic postlaminectomy membrane, if a patient requires a subsequent posterior operation, the exposed dura over the length of the laminectomy can make the revision operation unnecessarily more tedious, difficult, and risky to perform.

Skip laminectomy is a modified procedure that was designed to limit posterior muscle trauma and neck pain, with the promise of also limiting postoperative kyphosis. With this approach, two consecutive stenotic disc levels are decompressed via a standard laminectomy of the lamina between the stenotic levels, combined with a partial laminectomy of the lower adjacent vertebra. Thus, a C3-7 decompression can be achieved by laminectomy of $\mathrm{C} 4$ and $\mathrm{C} 6$, with partial laminectomies and flavum resection at other levels. At the "skipped" lamina (C3, C5, and C7 in this example), the muscular attachments to the spinous

Table 1. Potential clinical findings in cervical myelopathy*

\begin{tabular}{|c|c|}
\hline Symptoms & Signs \\
\hline $\begin{array}{l}\text { Hand clumsiness, difficulty with fine motor skills } \\
\text { (e.g., buttoning, jewelry, handwriting) }\end{array}$ & Motor Weakness (most commonly in the hands) \\
\hline $\begin{array}{l}\text { Diffuse, non-dermatomal upper Extremity Numbness } \\
\text { (usually the hands) }\end{array}$ & $\begin{array}{l}\text { Upper motor neuron signs: Hyper-reflexia, clonus, } \\
\text { babinski, scapulohumeral reflex }\end{array}$ \\
\hline Gait Instability, bumping into walls, feeling "drunken" or "wobbly" & Objective gait disturbance: timed walking tests \\
\hline May or may not have neck or arm pain & $\begin{array}{l}\text { Provocative signs: Hoffmann's, inverted } \\
\text { brachioradialis reflex }\end{array}$ \\
\hline $\begin{array}{l}\text { Lhermitte's Symptoms - electric "jolts" down the spine } \\
\text { with particular neck movements }\end{array}$ & Lhermitte's Sign \\
\hline \multirow[t]{2}{*}{ Bowel/ bladder incontinence } & Muscle wasting (most commonly the hand) \\
\hline & $\begin{array}{l}\text { Myelopathy hand: Finger Escape Sign, } \\
\text { inability to rapidly grip and release }\end{array}$ \\
\hline
\end{tabular}

* As a clinical diagnosis, it is important to note that many patients with cervical myelopathy may not present with all or even a majority of the above signs and symptoms. In addition, the absence of certain characteristic findings, such as hyperreflexia, in no way rules out the diagnosis of myelopathy. Rhee JM, Heflin JA, Hamasaki T, Freedman B: Prevalence of physical signs in cervical myelopathy: a prospective, controlled study. Spine 2009; 34: 890-895. 


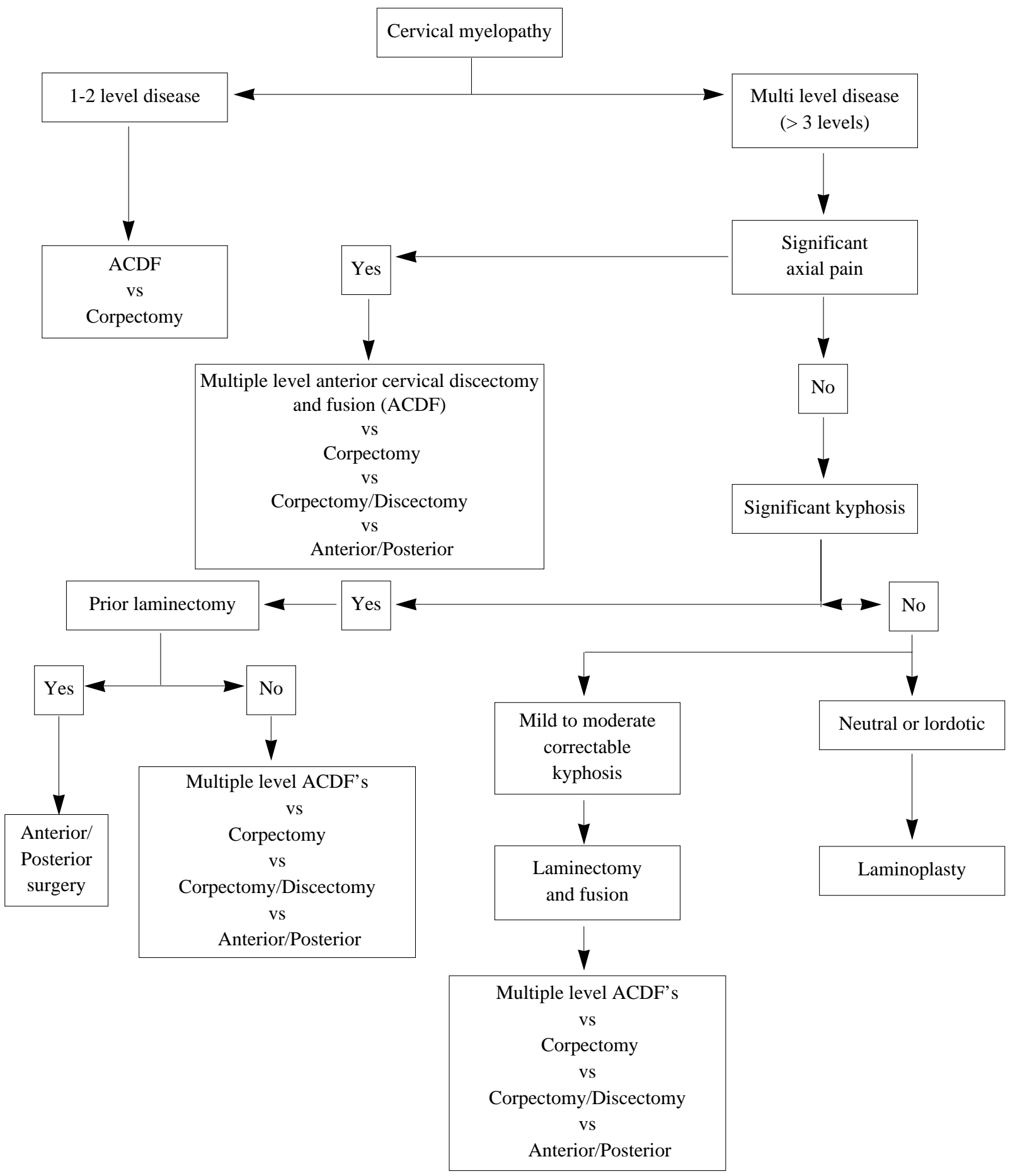

Fig. 1. General guidelines for surgical management of cervical myelopathy

processes are left intact, thereby helping to preserve sagittal alignment and limit post-laminectomy kyphosis. Two-year follow up data demonstrated similar neurologic outcomes as open door laminoplasty with less postoperative neck pain and better range of motion'. However, as the authors of the technique admit, this procedure may be better suited to patients with moderate stenosis or in whom ossification of the yellow ligament is the primary compressive lesion. If the stenosis is severe or continuous, as may be the case in congenital stenosis or extensive OPLL, skip laminectomy may provide suboptimal decompression of the cord. 


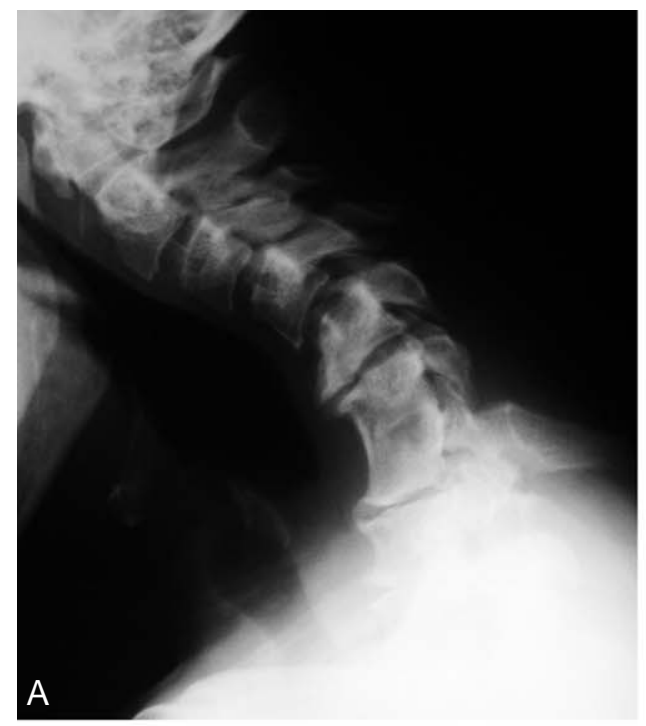

Fig. 2. Fifty-two year old woman with postlaminectomy kyphosis and myelopathy. (A) Several factors contribute to her kyphosis: 1) an anterior cervical discectomy without fusion at C6-7 in the remote past that healed in kyphosis; 2) severe disc degeneration at C5-6 and C7-T1; 3) iatrogenic spondylolisthesis at C4-5; and 4) multilevel laminectomy. (B and $\mathbf{C}$ ) AP and lateral xrays after C5 corpectomy, C3-4 ACDF, and posterior fusion performed to correct kyphosis and decompress the spinal canal.
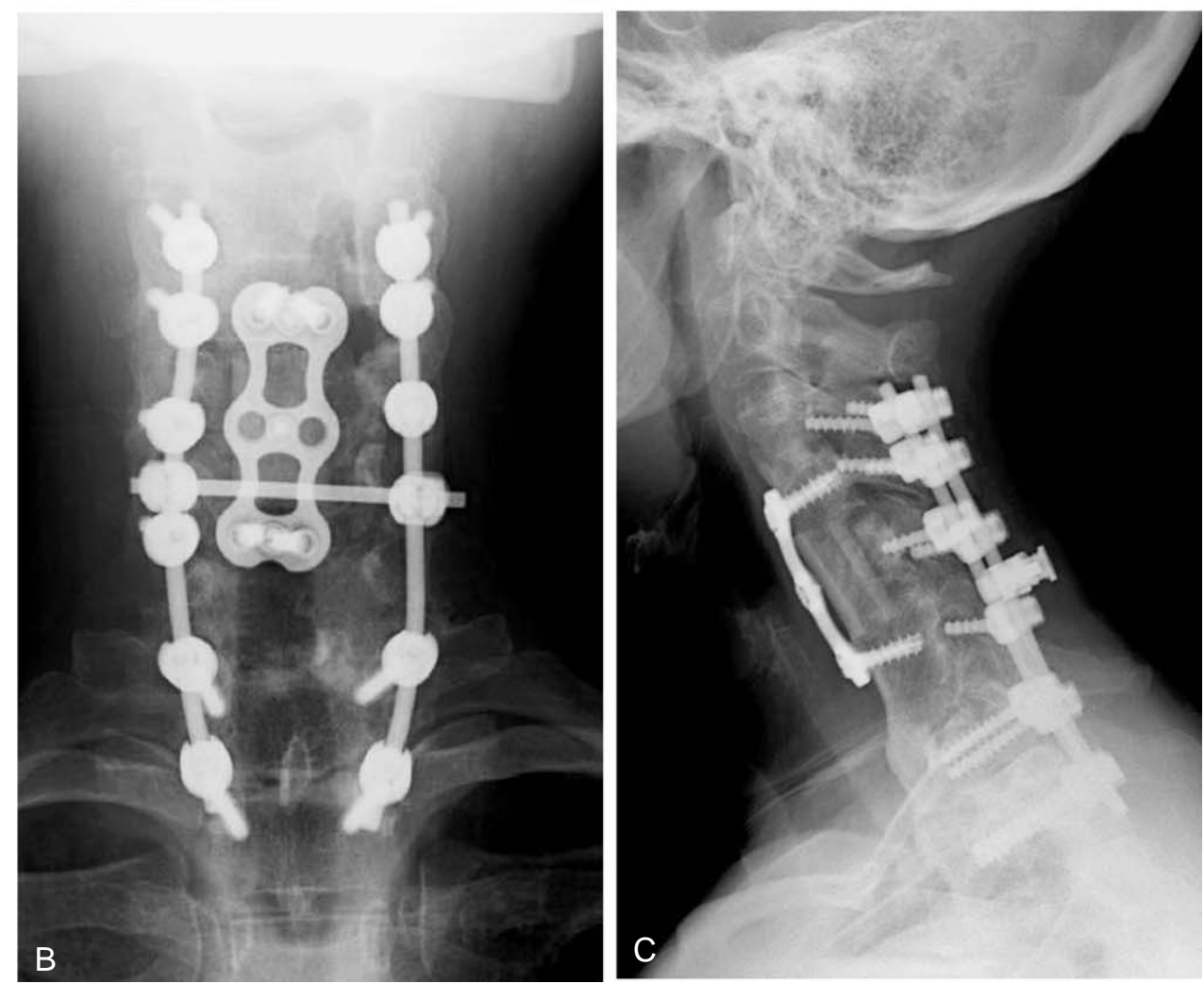

\section{Laminectomy with fusion}

In order to avoid some of the drawbacks associated with laminectomy alone, a posterior fusion can be added. Currently, fusion in association with laminectomy is typically performed with lateral mass screws. Autologous bone graft from the iliac crest is generally recommended to enhance fusion rates, but successful outcomes have been noted with the use of local autograft bone only $y^{8}$. Fusion may improve spondylotic neck pain and prevent post-laminectomy kyphosis. In addition, fusion may limit repetitive microtrauma to a healing cord and also prevent the development of instability which has been associated with poorer neurologic outcomes. In one study of laminectomy without fusion', patients who developed $2 \mathrm{~mm}$ flexion-extension instability or $2 \mathrm{~mm}$ more instability than was present preoperatively were noted to have slightly inferior neurologic outcomes.

Good outcomes have been reported by several authors with laminectomy and fusion for cervical myelopathy ${ }^{8,10}$. 
Indeed, in the properly selected patient who has enough lordosis to allow cord drift-back to occur after a posteriorlybased decompression, neurologic outcomes are likely to be similar regardless of the specific method of dorsal decompression (i.e., laminectomy, laminectomy and fusion, or laminoplasty), at least in the short to medium term, provided that significant instability or kyphosis do not develop. Differences among the methods are most likely to manifest in terms of complications. In a non-randomized comparison of laminoplasty versus laminectomy and fusion ${ }^{11}$, the complication rate heavily favored laminoplasty: fourteen complications arose in thirteen patients in the laminectomy and fusion group, compared with no complications in the laminoplasty group. The majority of complications were fusion related, such as nonunion, implant failure, adjacent segment degeneration, and substantial donor site pain.

In light of these findings, laminectomy and fusion with lateral mass instrumentation may be preferred in multilevel
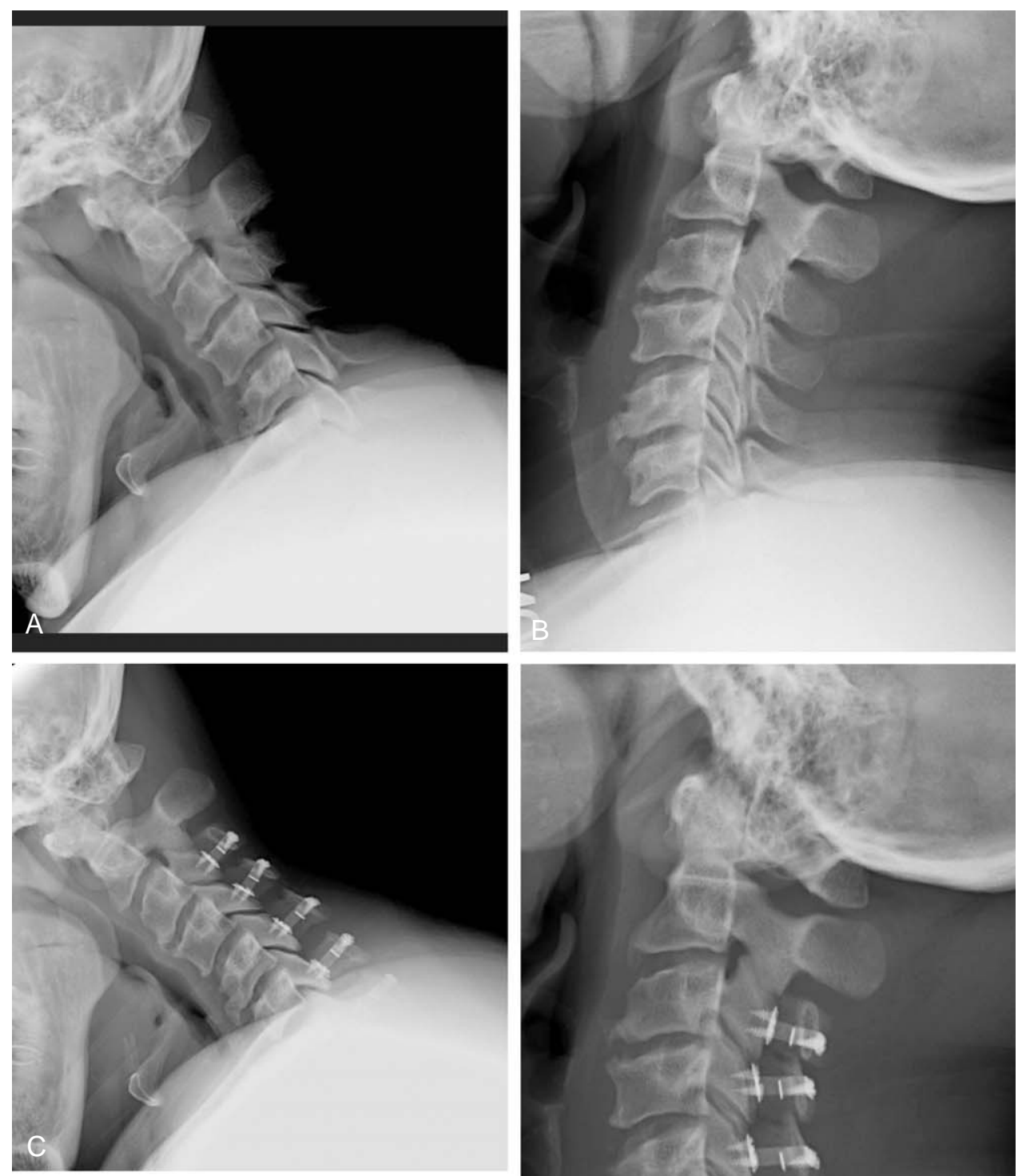

Fig. 3. Laminoplasty is a relatively motionpreserving procedure. Flexion-extension xrays before (A and $\mathbf{B})$ and at 3 months after ( $\mathbf{C}$ and D) multilevel laminoplasty demonstrate preservation of range of motion. In many cases, however, some loss of motion occurs after laminoplasty.

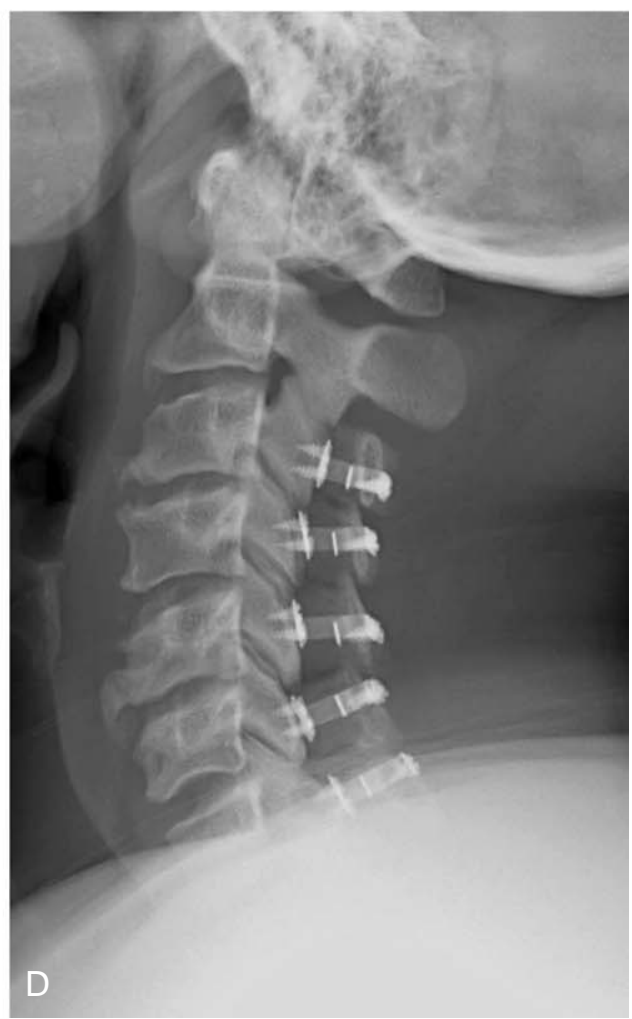


myelopathic patients with significant neck pain (e.g., from facet arthropathy) if one aim of surgery is to control the spondylotic neck pain with fusion. Because laminectomy and fusion better preserves sagittal alignment than does laminoplasty, it may also be preferred in patients who are not too kyphotic to be decompressed posteriorly (e.g., neutral to slightly kyphotic alignment), but stand a higher risk for progressing into kyphosis if a posterior decompression is performed without fusion. Mild to moderate amounts of flexible kyphosis can be corrected posteriorly after laminectomy prior to securing the instrumentation. However, for those in whom fusion is not necessary, laminoplasty may be a better approach.

\section{Laminoplasty}

Laminoplasty has several advantages over laminectomy alone as well as laminectomy and fusion. In contrast to laminectomy alone, laminoplasty better preserves cervical alignment. As opposed to laminectomy and fusion, laminoplasty is a relatively motion preserving procedure (Fig. 3). No fusion is required, but a fusion and instrumentation can be done in association with laminoplasty if desired. Thus, all fusion related complications can be avoided, including pseudarthrosis, donor site morbidity, and adjacent segment stress transfer. Fusions can be avoided in patients at high risk for pseudarthrosis, such as diabetics, the elderly, and chronic steroid users. However, laminoplasty in no way precludes the performance of a fusion. In fact, the preservation of dorsal elements with laminoplasty as opposed to laminectomy provides a much larger surface area for fusion to occur, which can be done with either the open door or French door variations. Finally, as mentioned previously, the preservation of a bony covering for the dural sac prevents the formation of post-laminectomy membranes that may impinge the cord as well as make any revision posterior approaches safer and easier to perform.

\section{Clinical evidence for laminoplasty}

The theoretical advantages of laminoplasty have been borne out in clinical trials versus multilevel anterior corpectomy. Yonenobu et al..$^{12}$ compared 42 patients who underwent laminoplasty versus 41 patients who had multilevel anterior corpectomy for cervical spondylotic myelopathy.
Neurologic outcomes were similar between the two groups, with both demonstrating good improvement in Japanese Orthopaedic Association myelopathy scores. However, the laminoplasty group demonstrated a significantly lower complication rate than the corpectomy group (7\% versus 29\%). The majority of complications as expected in the corpectomy group were graft related, including pseudarthrosis, graft displacement, and graft fracture. In the laminoplasty group, the only complications were three cases of $\mathrm{C} 5$ root paresis, all of which resolved. Edwards et al. ${ }^{13}$, also found similar neurologic outcomes between the two procedures, but again found a much lower complication rate in the laminoplasty group (1/13 patients versus $10 / 13$ patients). In this series, the majority of the complications with corpectomy were related to the anterior surgical approach (4 cases of persistent dysphagia, 2 cases of persistent dysphonia).

Laminoplasty is also a useful approach in patients who develop adjacent segment stenosis after previous anterior cervical fusions, in that it allows for multilevel decompression through virgin territory without the need to fuse additional levels and further predispose to accelerated wear at adjacent segments ${ }^{14}$. Microsurgical posterior herniotomy (i.e., discectomy) has also been described via laminoplasty approach to remove central and paracentral disc herniations ${ }^{15}$. Although the advocates of this procedure note excellent outcomes with no neurologic complications, it is generally not necessary to actually remove disc herniations in order to obtain satisfactory outcomes with laminoplasty. The canal expansion obtained with laminoplasty is such that the vast majority of disc herniations no longer remain symptomatically compressive lesions. In our experience, even disc herniations that impinge upon both the cord and the exiting root posterolaterally causing myeloradiculopathy can be rendered asymptomatic by laminoplasty with associated foraminotomy if necessary, whether or not the disc is actually excised. In the occasional patient with persistent radiculopathy or myelopathy after laminoplasty, focal anterior decompressions can be performed at any remaining levels of compression, saving on the overall number of levels fused.

\section{Potential issues with laminoplasty}

\section{Wound infections}

Deep wound infections occur in about 3 4\% of patients 
with laminoplasty and other posterior operations, which is substantially higher than the infection rate associated with anterior surgery $(<1 \%)$.

\section{Segmental root level palsy}

Laminoplasty is by no means a perfect operation and does have its disadvantages. Segmental root level palsy remains a significant concern. A common misconception is that $\mathrm{C} 5$ palsies are unique to laminoplasty, but in fact they can occur after laminectomy, laminectomy and fusion, and even anterior decompressions ${ }^{16}$. Its incidence after laminoplasty ranges from $5 \sim 12 \%{ }^{17}$ and most commonly affects the $\mathrm{C} 5$ root, although any root can be involved. The palsies tend to be motor-dominant, although sensory dysfunction and radicular pain are also possible. The problem may arise at any point postoperatively, from immediately to 20 days later $^{17}$, complicating what otherwise appeared to be a successful decompression of the spinal cord. Recovery to useful function usually occurs over weeks to months in the majority of patients, but has been reported to take as long as 6 years $^{18}$. The mechanism is unclear but is commonly thought to be associated with cord drift-back and subsequent stretching of the $\mathrm{C} 5$ root, which is more vulnerable than the other roots due to its short, direct course and the fact that its excursion after decompression may be greater than that of other roots because the C5 level is typically at the apex of cervical lordosis .

\section{Neck pain}

Laminoplasty has also been associated with postoperative neck pain. Because no arthrodesis is performed, laminoplasty is not a procedure designed to address painful spondylosis. The controversy centers on whether the neck pain reported with laminoplasty reflects new-onset postoperative symptoms or simply persistence of preoperative spondylotic pain. Hosono et al. ${ }^{19}$ performed open door laminoplasty and found postoperative axial symptoms in $60 \%$ of laminoplasty versus $19 \%$ of anterior fusion patients, a significant difference. In addition, $75 \%$ of those reporting postoperative neck and shoulder pain in the laminoplasty group had new onset pain. Kawaguchi et al. ${ }^{20}$ also performed open door laminoplasty and found a significant incidence of postoperative axial symptoms. In contrast, Yoshida et al. ${ }^{21}$ found that spinous process splitting laminoplasty did not affect either the development or resolution of axial neck and shoulder symp- toms. The exact etiology for the postoperative neck pain is unclear, but may be related to stiffening of the facet joints or denervation and injury to the nuchal musculature. In the author's own experience, new onset midline neck pain is relatively rare, although persistence or amplification of preoperative axial pain is common. Thus, laminoplasty is ideally suited to the patient with little to no axial pain.

It is also possible that the neck pain reported in the older laminoplasty literature may be related to the practices of prolonged postoperative immobilization as well as bone grafting of the hinge side. When these practices were abandoned and patients were encouraged to begin active rehabilitation of cervical extensor muscles early postoperatively, the incidence of axial neck symptoms was much lower ${ }^{22}$.

\section{Loss of motion}

Even when a laminoplasty is performed without fusion, loss of motion typically does occur. The cause may be multifactorial but may be related to facet joint injury with spontaneous fusion or alterations in tissue elasticity after an extensive posterior exposure. Prolonged postoperative immobilization may contribute to the problem. In addition, placing bone graft along the hinge side to assist in healing of the hinge may lead to undesired intersegmental fusion or stiffening. In a long term study of open door laminoplasty, Wada et al. ${ }^{23}$ reported $27 \%$ loss of range of motion $\left(37.1^{\circ}\right.$ preop to $27.1^{\circ}$ postop) in patients who were immobilized in a collar for only three weeks and who did not undergo bone grafting of the hinge side, compared with a $71 \%$ loss of motion $\left(40.2^{\circ}\right.$ preop to $11.6^{\circ}$ postop) in those who were immobilized for 2 3 months and were bone grafted on the hinge side. In general, about $30 \%$ loss of preoperative range of motion can be expected in the $\mathrm{C} 2-7$ motion arc, even with early mobilization

\section{Postoperative kyphosis}

Although the overall sagittal profile should be conducive to cord drift-back in order for laminoplasty to achieve satisfactory cord decompression, the absence of lordosis is not an absolute contraindication to laminoplasty. Suda et al. ${ }^{24}$ demonstrated that laminoplasty could be performed with acceptable neurologic recovery when the local kyphosis measured 13 degrees or less. In kyphotic patients demonstrating compressive lesions both anteriorly and posteriorly, laminoplasty may also achieve a direct decompressive 


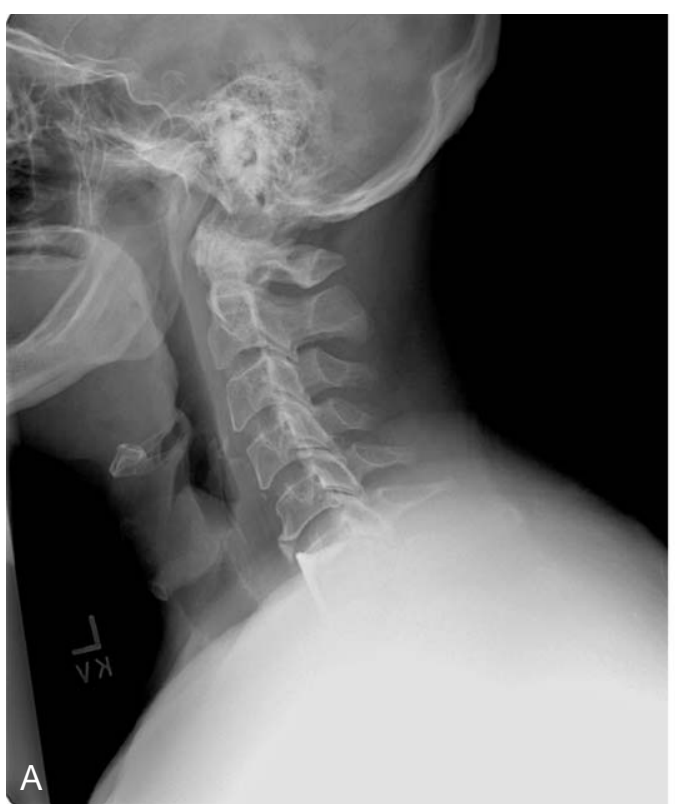

effect despite kyphosis.

However, even though laminoplasty can achieve decompression in the face of mild kyphosis and is not as kyphogenic a procedure as laminectomy alone, most patients do tend to lose some lordosis after laminoplasty (Fig. 4). Thus, laminoplasty is ideally indicated in the lordotic patient, and we do not routinely recommend laminoplasty in those who do not have at least neutral to slightly lordotic alignment preoperatively.

In our own experience as well as that reported in the literature, small amounts (grade I) of spondylolisthesis, on the other hand, do not appear to adversely affect outcomes after laminoplasty. One study of 67 patients found no differences in axial symptoms, neurologic recovery, or radiologic outcomes in patients with and without cervical spondylolisthe$\mathrm{sis}^{25}$. As noted previously, a selective fusion, instrumented or not, can be done along with laminoplasty at a listhetic segment if there is concern that it is symptomatic or that it might progress after decompression.

\section{Progression of OPLL}

In patients with severe OPLL and dural deficiencies, laminoplasty provides a safer, easier solution to decompression than does anterior corpectomy. However, because the OPLL is not resected with laminoplasty, the potential remains for growth and expansion of OPLL over time. In most cases, symptomatic regrowth at the decompressed levels is unlikely because the canal diameter is made so large that a modest amount of OPLL expansion will not cause

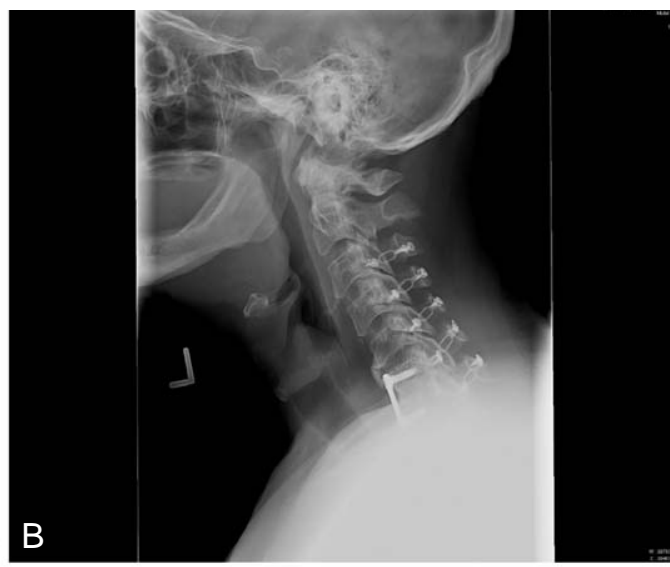

Fig. 4. Even with laminoplasty, some loss of lordosis may occur. Note in this patient, a subtle loss of lordosis postoperatively (A) versus preoperatively (B).

recurrent stenosis. But if the OPLL expands longitudinally to include previously undecompressed adjacent segments, symptomatic cord compression may occur. This scenario is more likely in younger patients with significant OPLL, and thus consideration should be given at the initial laminoplasty to prophylactically decompress segments adjacent to the limits of the OPLL, particularly the more cephalad area. For example, if such a patient has a large mass of OPLL arising at $\mathrm{C} 3$, with stenosis distal to $\mathrm{C} 3$ but not involving $\mathrm{C} 2-3$, one might still consider decompressing $\mathrm{C} 2$ prophylactically. Of course, the benefit of prophylactic decompression must be tempered against the possible morbidity of detaching the extensor attachments from $\mathrm{C} 2$ and abetting kyphosis.

\section{Open door laminoplasty: surgical technique}

There are many methods for performing laminoplasty, but the open and French door methods are the most common. The common theme in all variations of laminoplasty is the creation of a hinge at the junction of the lateral mass and lamina by thinning the dorsal cortex but not cutting completely through the ventral cortex, thereby allowing the creation of greenstick fractures. In the open door technique, the hinge is created unilaterally; in the French door version, the hinge is created bilaterally. The opening is performed on the opposite lateral mass-laminar junction in an open door procedure, or in the midline with the French door variation. Opening the laminoplasty increases the space available for the spinal cord, which drifts away from compressive lesions 

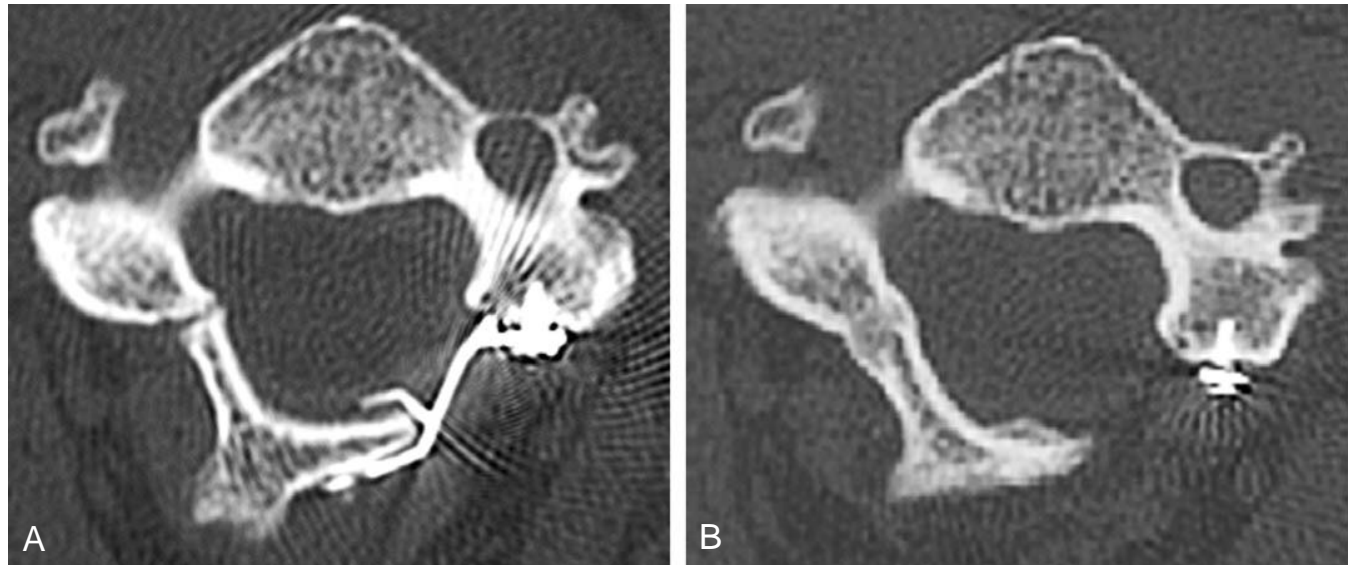

Fig. 5. Axial CT scans of a plated laminoplasty. (A) At 3 months postoperatively, note that the hinge was inadvertently made too "floppy", with both the dorsal and ventral cortices being cracked. (B) At 2 year follow up, however, the hinge has completely healed and remodeled. Plates provide the most stable form of laminoplasty fixation and are particularly helpful in cases such as these with "floppy" hinges.

into the space created. The opening can then be held patent with bone (e.g., autologous spinous process or rib allograft), sutures, suture-anchors, ceramic spacers, or specially designed plates ${ }^{26}$ (Fig. 5). Laminoplasty was initially designed in Japan, where it has enjoyed a long track record of success, but it is gaining wider acceptance in North America in light of proven benefits in the properly chosen patient. The open door method will be described here.

\section{Anesthetic concerns}

Excessive extension during intubation must be avoided in myelopathic patients, as extension diminishes the space available in the spinal canal. Awake, fiberoptic intubation should be considered in the severely myelopathic patient, particularly if the patient has a difficult airway and poorly tolerates extension (e.g., experiences Lhermitte's or other neurologic symptoms in extension). If an awake intubation is performed, a neurologic exam involving the motor function of all four extremities is ideally documented prior to putting the patient to sleep. When in doubt, it is preferable to err on the side of caution and perform a fiberoptic intubation.

Hypotensive anesthesia should also be avoided throughout the case in order to maintain spinal cord perfusion. Patients with cervical spondylotic myelopathy may have impairment of anterior spinal cord circulation due to compression from ventral OPLL. In the setting of already compromised cord perfusion, intraoperative drops in blood pressure can have catastrophic neurologic consequences. The anesthesiologist should be alerted to the importance of maintaining adequate blood pressure, and arterial lines may be recommended to allow for continuous monitoring of hemodynamic parameters, especially in patients who exhibit cardiovascular lability. Pressors, such as neosynephrine, are commonly needed intraoperatively. Although there are no established guidelines regarding where the blood pressure should be maintained, a systolic pressure of at least $100 \mathrm{mmHg}$, or keeping the patient relatively normotensive with respect to baseline, probably strike reasonable balances between the competing priorities of spinal cord perfusion versus excessive surgical site bleeding. Hypotension can occur during surgery for a number of reasons, but it is most commonly encountered during posterior cervical operations during prone, reverse trendelenberg positioning, prior to surgical stimulation. Therefore, it is important for the surgeon to alert the anesthesiologist to this possibility and be vigilant even prior to incision.

\section{Spinal cord monitoring}

Spinal cord monitoring in the form of somatosensory evoked potentials (SSEP) or motor evoked potentials (MEP) is recommended when operating on patients with myelopathy. In severely myelopathic patients with extremely tight stenosis, one may consider obtaining a set of monitoring data after intubation but before positioning to serve as a baseline for comparison to ensure safe positioning. Monitoring may also help prevent brachial plexopathies from positioning, mainly due to excessive longitudinal ten- 
sion from taping the shoulders, especially in larger individuals. Although controversy also exists as to the necessity of obtaining motor evoked potentials (MEPs) during cervical spine surgery ${ }^{27,28}$, currently available data indicate that if motor evoked potentials are available, they may provide useful adjunctive information to that obtained through SSEPs $^{29}$. Furthermore, SSEPs only monitor the function of the dorsal columns and extrapolates their function onto the function of the cord as a whole. In patients with cervical spondylotic myelopathy or OPLL, where ventral cord compression typically predominates, it is theoretically preferable to directly monitor anterior motor column function instead.

\section{Positioning}

Proper positioning is critical when operating on myelopathic patients. It prevents iatrogenic neurologic injury from excessive extension, limits bleeding, and makes the surgery technically easier to perform. There are four elements to a properly positioned patient for laminoplasty (Fig. 6). First, cervical tongs are used to rigidly immobilize the cervical spine and prevent pressure on the eyes and face. Second, longitudinal bolsters placed along the lateral edges of the chest and abdomen prevent abdominal compression and thus increased venous pressure and bleeding. Third, reverse trendeleburg positioning also decreases the venous pressure head to the cervical spine. As mentioned above, however, care must be taken to monitor for hypotension in this position. Fourth, the cervical spine should be placed in a neutral to slightly flexed alignment. The amount of preoperative extension tolerated by the patient without neurologic symptoms should be assessed and not exceeded during positioning. In addition to potential neurologic sequelae, excessive extension may make laminoplasty technically more difficult to perform because of the increased overlap or "shingling" between adjacent laminae which results with relative neck extension versus flexion (Fig. 7). In the shingled position, both the open and hinge sides are harder to complete as the caudal end of the superior lamina overlaps the cephalad portion of the inferior lamina. Furthermore, overlap of $\mathrm{C} 2$

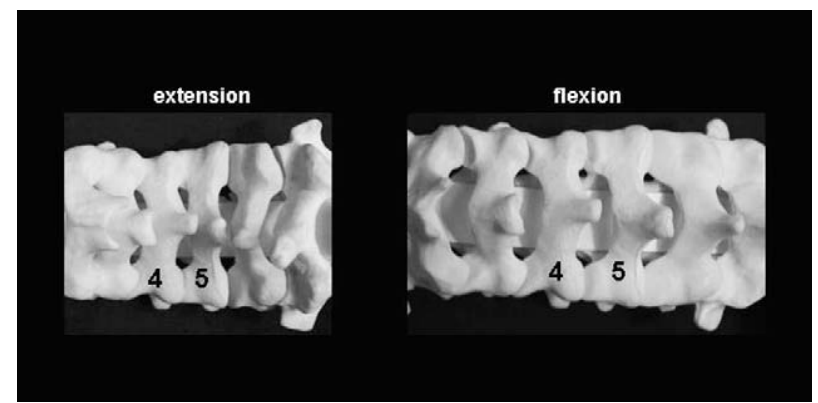

Fig. 7. In extension (left), there is greater overlap or "shingling" of the adjacent laminae, making surgery more difficult to perform. In relative flexion (right), there is less overlap, but greater care must be taken during exposure to avoid plunging into the canal. A relatively flexed posture will also tend to increase spinal canal dimensions and thus be neurologically safer in those who have severe stenosis and worsening of neurologic symptoms in extension.

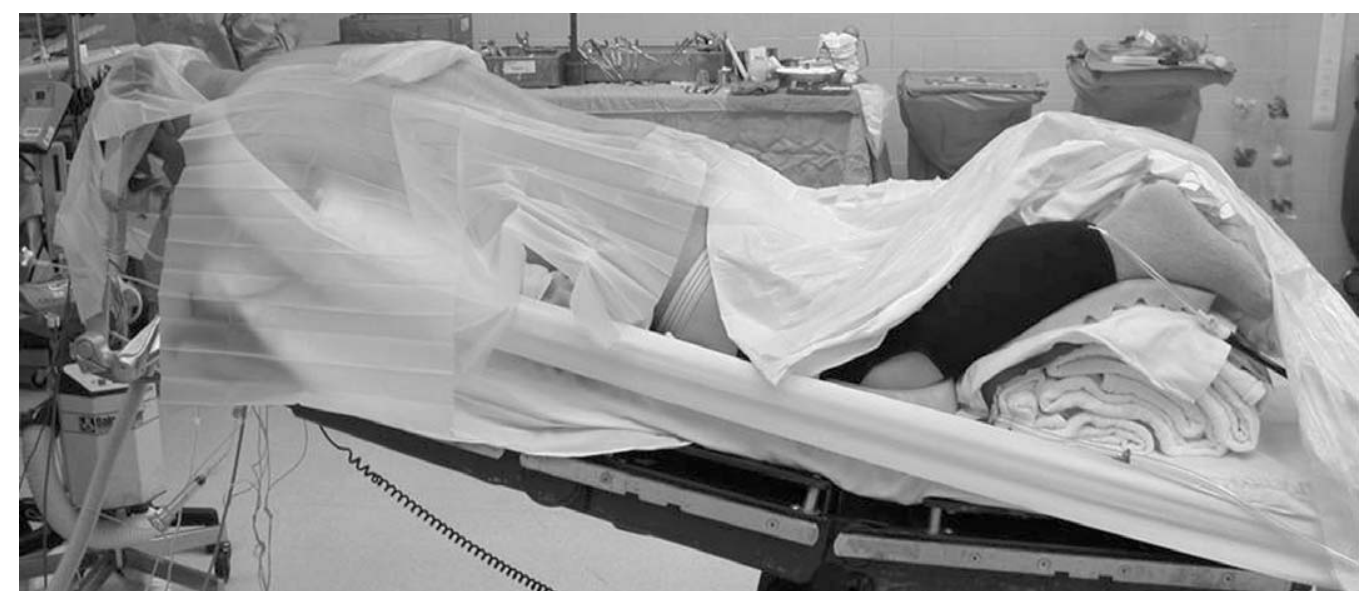

Fig. 6. There are four elements of a properly positioned patient for posterior cervical surgery. 1) Tongs are used to suspend the eyes and face from pressure, 2) the bed is placed into reverse trendelenberg to decrease venous pooling at the surgical site, 3) longitudinal bolsters (not seen) running laterally along the thoracoabdominal regions also decrease abdominal pressure and thus the venous pressure head at the surgical site, and 4) the neck is placed into a neutral to slightly flexed posture in order to decrease inter-laminar "shingling" or overlap. If a fusion is performed, the neck should be placed into a more lordotic postion prior to locking down the instrumentation. 


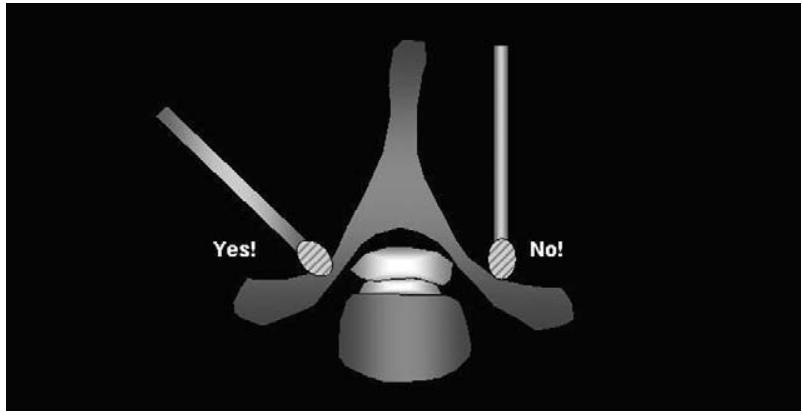

Fig. 8. Burr orientation: When creating either the hinge or open sides, the burr should be placed at the lateral mass-laminar junction and then oriented perpendicular to the lamina (left). The burr should not be oriented perpendicular to the floor (right), as this will lead to unnecessary removal of facet and make it more difficult to achieve the goal of entering and decompressing the spinal canal.

on $\mathrm{C} 3$ requires more aggressive resection of the inferior portion of $\mathrm{C} 2$ to allow $\mathrm{C} 3$ to open on $\mathrm{C} 2$. If a fusion is also performed, the neck can be extended after decompression into a more lordotic position for the fusion.

\section{The surgical procedure}

In order to limit muscle bleeding and perioperative neck pain, it is important to stay in the midline raphe during the approach to the spinous process, then maintain a strict subperiosteal plane as dissection proceeds laterally. As much as possible, the nuchal attachments onto the $\mathrm{C} 2$ spinous process should be preserved. Some advocate, if necessary, osteotomizing the spinous process with the muscular attachments in order to facilitate later reattachment and prevention of postoperative kyphosis ${ }^{30}$. The facet joints should be preserved-only the medial aspect of the joint needs to be exposed.

After exposure and confirmation of levels, the open side is created first using a high speed burr, generally on the side of greatest compression or clinical severity. A high speed burr is used to remove the posterior cortex at the junction of the lateral mass with the lamina. If burring is performed too medially, there may be a portion of the spinal cord which is not uncovered after opening the hinge. If burring occurs too laterally, the surgeon will enter the lateral mass rather than the spinal canal and not be able to open the lamina. Burring should be done at approximately a $45^{\circ}$ angle, perpendicular to the lamina, so that the burr enters the canal rather than burrowing into the lateral mass (Fig. 8). The burr is used to

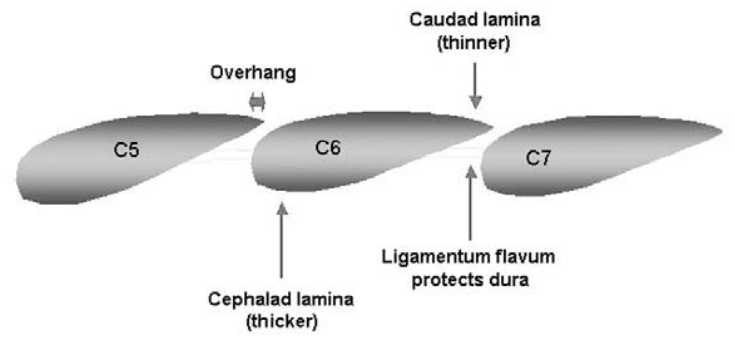

Fig. 9. Laminar anatomy. A sagittal representation of the lateral mass-laminar junction, where both the hinge and open sides of the laminoplasty are created. Note that the cepahalad portion of the lamina is 1) thicker than the caudal end, 2) covered by an "overhang" from the lamina above (dependent in part on the amount of flexion during positioning), and 3) not protected on its ventral surface by ligamentum flavum. For these reasons, burring is more difficult on the cephalad versus caudad portion of the lamina. If the laminoplasty fails to open, inspect the cephalad portion of the lamina for inadequate thinning. Note also that relative flexion during positioning will decrease the amount of inter-laminar overhang, making surgery easier to perform.

thin the lamina to a flake of anterior cortex. Over the caudal third to half of the lamina, the yellow hue of the ligamentum flavum will become evident as the anterior cortex of the lamina is appropriately thinned. Over the cephalad portion of the lamina, the blue hue of epidural vein or dura can be seen. At this point, a microcurette or microkerrison rongeur can be used to remove the remaining bone and flavum. If burring is done properly, the opening can be created with minimal intrusion into the spinal canal. Epidural bleeding may be encountered at this point and can readily be controlled with the application of bipolar cautery or thrombin-gelfoam. In general, thinner patients tend to bleed less, and proper positioning also limits bleeding.

Next the hinge is created. The hinge is located at the mirror image location to the opening, namely, at the junction of the lamina with the lateral mass on the less symptomatic side. In order to preserve the "springiness" of the hinge, aggressive removal of bone from the hinge side should be avoided. Generally, the dorsal cortex is removed and the ventral cortex is thinned but not completely removed. Performing the hinge after the opening allows the surgeon to continually test the springiness of the hinge and remove only enough bone to achieve adequate opening. The thickest portion of the lamina is always at the cephalad end (Fig. 9). Furthermore, because of the shingling effect, the cephalad portion of the caudal lamina is always covered by the 
overhang of the caudal portion of the cephalad lamina. Therefore, if the hinge fails to give or the open side fails to open, it is almost always due to inadequate cortical bone removal at the cephalad portion of the lamina

Once the lamina at each level has been cut, the C2-3 and C7-T1 interspinous ligaments are resected. The surgeon then firmly but gently pushes on the spinous processes from the open side, creating greenstick fractures on the hinged side. Enough plastic deformation is created in order to place approximately 10 12 mm grafts or plates. Excessive opening of the laminoplasty is not necessary and may be associated with excessive cord drift back and potentially higher rate of segmental root palsies as a result. As the laminoplasty is partially opened, the ligamentum flavum is placed under tension and can be sharply divided with a kerrison rongeur between $\mathrm{C} 2-3, \mathrm{C} 7-\mathrm{T} 1$, and the intervening segments from C3-7. After the laminoplasty is opened, dural pulsations can often be seen. Ideally, each lamina should open with a "springy" sensation, and complete fractures on the hinge side should be avoided if possible, especially if bone struts are used to keep the laminoplasty open, because the struts will not lock in as firmly.

There are a variety of options to keep the laminoplasty open. Rib struts are a classic method and are fashioned like an " $\mathrm{H}$ " with grooves that lock in to the lateral mass on one side and the cut edge of the lamina on the other. The tension on the greenstick fracture keeps the struts in place, and typically no supplemental fixation is necessary. Classically, three struts placed at $\mathrm{C} 3, \mathrm{C} 5$, and $\mathrm{C} 7$ are used. Alternatively, sutures, suture anchors, or mini-plates can be used to keep the laminoplasty open. Currently, we prefer to use mini plates because they are the easiest to use and, because they are more stable, tend to be more forgiving of loose hinges should they occur. The plates are unlikely to dislodge. Although screw back out may occur infrequently, it is generally of no consequence as long as the plate is securely fitted at the time of surgery and the hinge side eventually heals with either a fibrous or bony union. The optimal number of levels to plate is unclear. We prefer to plate each level in order to provide greatest stability to the construct. During placement of the plates, care should be taken to avoid violating the subjacent facet with the screws, particularly in $\mathrm{C} 7$ where the lateral mass can be quite thin. Regardless of the method used, the objective is to keep the laminoplasty open until the greenstick fracture heals on the hinge side. Premature closure of the laminoplasty may result in inadequate spinal cord decompression.

\section{Conclusions}

Posterior approaches to spinal cord decompression have their greatest advantage over anterior surgery in patients with multilevel myelopathy involving three or more motion segments. Among the posterior approaches, laminoplasty is best in those who do not need a fusion. If necessary, fusion can be done in conjunction with laminectomy or laminoplasty and can better preserve lordosis but at the consequence of greater limitation of motion and potential fusion/ instrumentation-related complications. Although unresolved or new onset neck pain and segmental root level palsy can be issues with laminoplasty, the low overall incidence of complications and avoidance of routine fusion make laminoplasty an attractive option in the appropriate patient with multilevel myelopathy. This is particularly true in patients who have little spondylotic neck pain and are lordotic. If the sagittal MRI suggests that a posterior approach will not allow adequate drift back and decompression of the cord, an anterior procedure should be considered instead.

\section{REFERENCES}

1. Geck MJ, Eismont FJ: Surgical options for the treatment of cervical spondylotic myelopathy. Orthop Clin North Am 2002; 33: 329-348.

2. Hirabayashi K, Bohlman HH: Multilevel cervical spondylosis. Laminoplasty versus anterior decompression. Spine 1995; 20: 1732-1734.

3. Mikawa Y, Shikata J, Yamamuro T: Spinal deformity and instability after multilevel cervical laminectomy. Spine 1987; 12: 6-11.

4. Kato Y, Iwasaki M, Fuji T, Yonenobu K, Ochi T: Longterm follow-up results of laminectomy for cervical myelopathy caused by ossification of the posterior longitudinal ligament. J Neurosurg 1998; 89: 217-223.

5. Matsunaga S, Sakou T, Nakanisi K: Analysis of the cervical spine alignment following laminoplasty and laminectomy. Spinal Cord 1999; 37: 20-24.

6. Morimoto T, Ohtsuka H, Sakaki T, Kawaguchi M: Postlaminectomy cervical spinal cord compression demonstrated by dynamic magnetic resonance imaging. Case report. J Neurosurg 1998; 88: 155-157.

7. Shiraishi T, Fukuda K, Yato Y, Nakamura M, Ikegami T: Results of skip laminectomy-minimum 2-year follow-up 
study compared with open-door laminoplasty. Spine 2003; 28: 2667-2672.

8. Huang RC, Girardi FP, Poynton AR, Cammisa Jr FP: Treatment of multilevel cervical spondylotic myeloradiculopathy with posterior decompression and fusion with lateral mass plate fixation and local bone graft. J Spinal Disord Tech 2003; 16: 123-129.

9. Guigui P, Benoist M, Deburge A: Spinal deformity and instability after multilevel cervical laminectomy for spondylotic myelopathy. Spine 1998; 23: 440-447.

10. Sekhon LH: Posterior cervical decompression and fusion for circumferential spondylotic cervical stenosis: review of 50 consecutive cases. J Clin Neurosci 2006; 13: 23-30.

11. Heller JG, Edwards CC 2nd, Murakami H, Rodts GE: Laminoplasty versus laminectomy and fusion for multilevel cervical myelopathy: an independent matched cohort analysis. Spine 2001; 26: 1330-1336.

12. Yonenobu K, Hosono N, Iwasaki M, Asano M, Ono K: Laminoplasty versus subtotal corpectomy. A comparative study of results in multisegmental cervical spondylotic myelopathy. Spine 1992; 17: 1281-1284.

13. Edwards CC 2nd, Heller JG, Murakami H: Corpectomy versus laminoplasty for multilevel cervical myelopathy: an independent matched-cohort analysis. Spine 2002; 27: 1168-1175.

14. Matsumoto M, Nojiri K, Chiba K, Toyama Y, Fukui Y, Kamata M: Open-door laminoplasty for cervical myelopathy resulting from adjacent-segment disease in patients with previous anterior cervical decompression and fusion. Spine 2006; 31: 1332-1337.

15. Sasai K, Saito T, Ohnari H, et al: Microsurgical posterior herniotomy with en bloc laminoplasty: alternative method for treating cervical disc herniation. J Spinal Disord Tech 2005; 18: 171-177.

16. Atkinson JH, Slater MA, Williams RA, et al: A placebocontrolled randomized clinical trial of nortriptyline for chronic low back pain. Pain 1998; 76: 287-296.

17. Uematsu Y, Tokuhashi Y, Matsuzaki H: Radiculopathy after laminoplasty of the cervical spine. Spine 1998; 23: 2057-2062.

18. Satomi K, Nishu Y, Kohno T, Hirabayashi K: Longterm follow-up studies of open-door expansive laminoplasty for cervical stenotic myelopathy. Spine 1994; 19: 507510.

19. Hosono N, Yonenobu K, Ono K: Neck and shoulder pain after laminoplasty. A noticeable complication. Spine 1996;
21: 1969-1973.

20. Kawaguchi Y, Matsui H, Ishihara H, Gejo R, Yoshino O: Axial symptoms after en bloc cervical laminoplasty. J Spinal Disord 1999; 12: 392-395.

21. Yoshida M, Tamaki T, Kawakami M, et al: Does reconstruction of posterior ligamentous complex with extensor musculature decrease axial symptoms after cervical laminoplasty? Spine 2002; 27: 1414-1418.

22. Kawaguchi Y, Kanamori $\mathbf{M}$, Ishiara $\mathbf{H}$, Nobukiyo $\mathbf{M}$, Seki S, Kimura T: Preventive measures for axial symptoms following cervical laminoplasty. J Spinal Disord Tech 2003; 16: 497-501.

23. Wada E, Suzuki S, Kanazawa A, Matsuoka T, Miyamoto S, Yonenobu K: Subtotal corpectomy versus laminoplasty for multilevel cervical spondylotic myelopathy: a long-term follow-up study over 10 years. Spine 2001; 26: 1443-1447.

24. Suda K, Abumi K, Ito M, Shono Y, Kaneda K, Fujiya M: Local kyphosis reduces surgical outcomes of expansive open-door laminoplasty for cervical spondylotic myelopathy. Spine 2003; 28: 1258-1262.

25. Kawakami M, Tamaki T, Ando M, Yamada H, Matsumoto T, Yoshida M: Preoperative instability does not influence the clinical outcome in patients with cervical spondylotic myelopathy treated with expansive laminoplasty. J Spinal Disord Tech 2002; 15: 277-283.

26. Park AE, Heller JG: Cervical laminoplasty: use of a novel titanium plate to maintain canal expansion--surgical technique. J Spinal Disord Tech 2004; 17: 265-271.

27. Darden BV 2nd, Hatley MK, Owen JH: Neurogenic motor evoked-potential monitoring in anterior cervical surgery. J Spinal Disord 1996; 9: 485-493.

28. Gokaslan ZL, Samudrala S, Deletis V, Wildrick DM, Cooper PR: Intraoperative monitoring of spinal cord function using motor evoked potentials via transcutaneous epidural electrode during anterior cervical spinal surgery. J Spinal Disord 1997; 10: 299-303.

29. Sethuraman V, Hilibrand A, Schwartz D: The utility of transcranial motor evoked potential monitoring as an indicator of impending cervical spinal cord injury. 29th Annual Meeting of Cervical Spine Research Society 2001. Monterey, CA.

30. Yonenobu K, Yamamoto T, Ono K: Laminoplasty for myelopathy: indications, results, outcome, and complications. (in Clark $\mathrm{C}$ ed. The cervical spine. 3rd ed. Philadelphia, Lippincott-Raven: 849-864, 1998). 